Acknowledgements: This work was supported by a grant from the Canadian Rheumatology Association through the Canadian Initiative for Outcomes in Rheumatology Care (CIORA).

Disclosure of Interest: None declared

DOI: 10.1136/annrheumdis-2017-eular.2007

\section{THU0630 OPIOID USE IN PATIENTS WITH POLYMYALGIA RHEUMATICA}

M.D. Richter $^{1}$, S.J. Achenbach ${ }^{2}$, J.A. Zamora-Legoff ${ }^{3}$, C.S. Crowson ${ }^{2}$, E.L. Matteson ${ }^{3}$. ${ }^{1}$ Graduate Medical Education; ${ }^{2}$ Division of Biomedical Statistics and Informatics; ${ }^{3}$ Division of Rheumatology, Mayo Clinic, Rochester, United States

Background: Polymyalgia rheumatic (PMR) is a systemic rheumatic inflammatory disease characterized primarily by musculoskeletal pain and stiffness. Glucocorticoid treatment is the current standard of care however the need for additional pain management, in particular the need for opioid therapy, has not been studied. Objectives: To examine the trends of chronic opioid use in patients with PMR over an 11 year period in Olmsted County, Minnesota, USA and compare this to subjects without the disease.

Methods: Retrospective data on opioid prescriptions were collected from 2005 to 2015 in a population-based incidence cohort of patients meeting the 2012 American College of Rheumatology classification criteria for PMR alongside comparison subjects. Poisson regression methods were used to compare opioid use between these groups.

Results: 244 patients with PMR and 211 non-PMR comparator subjects were included in the study. Rates of chronic opioid use were not significantly different between the two groups. $7.5 \%$ of patients with PMR were identified as chronic users by the end of the study period compared with $5.2 \%$ of non-PMR subjects. Any opioid use was also not significantly higher in PMR, with relative risk of $1.10(95 \% \mathrm{Cl} 0.97,1.26, \mathrm{p}=0.14)$. There were higher rates of chronic use among patients over 80 years in both groups.

Conclusions: PMR does not appear to be associated with increased rates of opioid use when compared with the general population.

Acknowledgements: This study was made possible by the Rochester Epidemiology Project, which receives support from the National institute on Aging of the $\mathrm{NIH}$.

Disclosure of Interest: None declared

DOI: 10.1136/annrheumdis-2017-eular.1237

\section{THU0631 DIRECT FINANCIAL BURDEN OF RHEUMATOID ARTHRITIS ON PATIENTS' LIFE IN A DEVELOPING NATION OF PAKISTAN, ONE YEAR PROSPECTIVE STUDY}

M.S. Ahmad. Orthopedic Surgery, Fazle Omar Hospital, Chenab Nagar, Pakistan

Background: Rheumatoid Arthritis is chronic inflammatory disease. Early diagnosis and management is necessary to ovoid joint destruction and to minimize disability. It effects 0.5 to 1 percent population. The female to male ratio is 3 to 1 . Health care cost is of great concern to patients, physicians and health care policy makers. The financial impact of rheumatoid arthritis treatment like any chronic illness is of great significance in developing society like Pakistan where 30 percent population lives below poverty line and annual per capita incomes are very low. Whereas prevalence is same with high medicines prices with poorly developed health insurance system and government funded hospitals are scanty.

Most of times attention in the field of health economy is focused on direct medical in general and hospital cost in particular but however there are some social societal customs which also increases the burden of direct cost. The direct cost includes expenditures like physician visit cost, diagnostic tests, medications. There are also some hidden charges to this direct cost which are not included in studies like transport charges, food bills during hospital stay and accompany person, female are companied by males. All included puts strain on economy and major share of annual income of patient is paid on management of disease. The worst scenario is when patient stops treatment and if lucky gets support from other sources like patient welfare societies or from relatives.

Objectives: To assess the direct cost of patient's every visit to hospital outpatient department prospectively for one year and total cost of one year was summed up and percentage to annual income spend on treatment was calculated.

Methods: Study carried out from January to December 2015 at Fazle Omar Hospital Rabwah Chenab Nagar and 150 patients either newly diagnosed or already on treatment of rheumatoid Arthritis were included. Data collected for next 1 year for each visit, patients with any other disease along with rheumatoid were excluded. The estimated annual income of patient or the person bearing the expenses was recorded before start of study. Every time patient visit hospital outpatient department, prescription copy kept and cost of medicines, laboratory investigations and consultation fee is calculated and a questionnaire is filled how much it cost coming hospital and going back home and during hospital stay on any other things. The total cost of every visit was recorded for one year.

Results: The mean total per patient income after conversion from local currency was 3000 US dollars against 1474 US dollars per capita income in 2015 . The annual average cost per patient including consultation fee, medicines purchased, laboratory investigations and other overhead expenses like transportation of patient and accompanied person and food bills during hospital stay was 1194 US dollars.
$41 \%$ of patient gross income was spend on management of rheumatoid arthritis. Investigations cost $12 \%$, medicines purchased $16 \%$, consultation $2 \%$ and over head visit charges cost $10 \%$ of the total mean of per patient annual income.

Conclusions: RA management consumes large portion of patients annual income and it has significant burden on developing world economy. References:

[1] The economic burden of rheumatoid arthritis in a developing nation: result from one year prospective cohort study in Thailand, Osiri M, Maetzel A, Tugwell P. J Rheumatol 2007 Jan.34(1):57-63.

Disclosure of Interest: None declared

DOI: 10.1136/annrheumdis-2017-eular.5571

\section{THU0632 VALIDATION OF INTERNET-BASED REPORTING OF PATIENT REPORTED OUTCOMES WITHIN THE SWEDISH RHEUMATOLOGY QUALITY REGISTER}

O.E. Hofstedt ${ }^{1}$, D. Di Giuseppe ${ }^{2}$, G.-M. Alenius ${ }^{1}$, N. Stattin ${ }^{3}$

$\mathrm{H}$. Forsblad-d'Elia ${ }^{1}$, L. Ljung ${ }^{1}$. ${ }^{1}$ Department of Public Health and Clinical Medicine, Umeå University, Umeå: ${ }^{2}$ Department of Medicine Solna, Karolinska Institutet, Stockholm; ${ }^{3}$ Swedish Rheumatism Association, Sundsvall, Sweden

Background: Previous studies have validated the use of clinic based touchscreens for registering patient reported outcome measures (PROMs) (1-3). The Swedish Rheumatology Quality (SRQ) register has implemented an internetbased method (PER (Patientens Egen Registrering, or Patients' sElf Registration)) for collecting PROMs.

Objectives: The aim of this study was to investigate the feasibility of the internet-based method as well as the validity of reported outcomes and disease activity scores compared to the gold standard paper format.

Methods: We recruited patients $(n=44$, mean age $=51.0$, standard deviation $=13.2$ years, $69.6 \%$ women) included in SRQ with a diagnosis of rheumatoid arthritis, psoriatic arthritis, juvenile arthritis, spondyloarthritis or ankylosing spondylitis. Before a planned visit at the rheumatology clinic the patients registered Visual Analog Scales (VAS) for global health, pain and fatigue, both electronically by PER and on paper. Patients with axial disease also registered BASDAI and BASFI related variables $(n=6)$. For patients with peripheral arthritis $(n=38)$, DAS28 was calculated using both methods. The differences between the methods were compared by T-test and Intra-class correlation (ICC). Agreement was visualized using Bland-Altman plots for all VAS registrations. The patients also answered a questionnaire regarding the used device and preferred method.

Results: No differences between PER or paper based VAS scores were found for VAS Global, VAS Pain and VAS Fatigue $(p=0.086, p=0.691$ and $p=0.197$, respectively). ICC scores ranged from 0.930 to 0.971 . Bland-Altman plots for VAS assessments showed good agreement and no proportional bias was detected (Fig 1). Mean difference for DAS28 was - 0.04 ( $p=0.177$ ). Of the recruited patients, $78 \%$, preferred the Internet based method. BASDAI and BASFI could not be evaluated due to a limited number of observations.

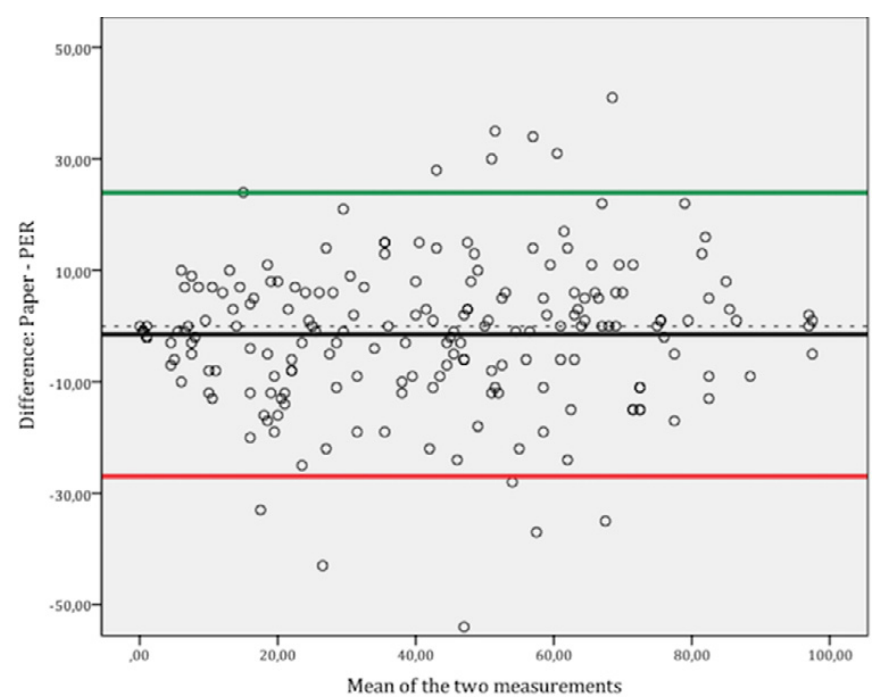

Fig. 1 Bland-Altman plot showing level of agreement of all VAS (VAS Global, VAS Pain, VAS Fatigue, VAS included in BASFI and BASDAI) between paper and PER method. BASDAI and BASFI scores multiplied by 10 for comparability.

Conclusions: Internet based reporting of PROMs supply valid VAS data. DAS28 scores from the internet-based method presents an acceptable alternative to the traditional paper formats.

\section{References:}

[1] Greenwood MC, et al. Rheumatology (Oxford). 2006

[2] Schefte DB, et al., Rheumatology (Oxford). 2010.

[3] Cunha-Miranda L, et al Rheumatol Int. 2015 
Disclosure of Interest: None declared

DOI: 10.1136/annrheumdis-2017-eular.2590

\section{THU0633 TEMPERATURE SENSITIVITY IN PATIENTS WITH RHEUMATOID ARTHRITIS}

P. Mandl ${ }^{1}$, F. Alasti ${ }^{1}$, A. Kerschbaumer ${ }^{1}$, R. Kaltenberger ${ }^{2}$, T. Krennert ${ }^{2}$, G.M. Supp ${ }^{1}$, U. Landesmann ${ }^{1}$, J.S. Smolen ${ }^{1}$, D. Aletaha ${ }^{1} .{ }^{1}$ Department of Rheumatology, Medical University of Vienna; ${ }^{2}$ Central Institute for Meteorology and Geodynamics, Vienna, Austria

Background: Studies evaluating weather sensitivity among patients with rheumatoid arthritis (RA) have yielded conflicting results.

Objectives: To evaluate whether patients with RA exhibit sensitivity to outside temperature.

Methods: We assessed correlation between mean daily temperature and selfreported pain (by visual analogue scale), and patient's global assessment of disease activity (PGA). Assessments documented in the RA database of our department as well as the average temperature obtained from the Central Institute for Meteorology and Geodynamics, were matched on a daily basis for a period of 10 years between 2005 and 2015 and analyzed using generalized estimating equation (GEE) and a mixed model analysis (MM). Patients with $<5$ visits in the study period, or with $<1$ visit/quarter or with pain $=0$ in $\geq 3$ consecutive visits and those living outside of the catchment area were excluded. Overlap between responsiveness of pain or PGA to temperature was calculated by Cohen's kappa. Results: A total of 399 patients with RA (average disease duration at first visit: $6.0 \pm 7.6$ years, average age: $57.7 \pm 13.9$ years, $82 \%$ female, mean CDAI $19.7 \pm 11.5$, $59.9 \%$ rheumatoid factor positive) were analyzed. Lower temperatures correlated significantly with higher pain levels (estimate: $-0.07, p=0.021$ ) in GEE, however the effect size was very small. When we performed MM with temperature as independent variable and VAS pain or PGA as dependent variable, the majority of patients showed no sensitivity to temperature, however $22 \%$ of patients were significantly sensitive to cold temperature with an estimate of $-0.29(p<0.0001)$ for pain and $-0.21 \quad(p=0.0005)$ for PGA (Figure 1). When we evaluated whether patients who demonstrate temperature-sensitivity to pain also exhibit temperaturesensitivity to PGA, we found an excellent overlap between the two patient groups (kappa: 0.81).

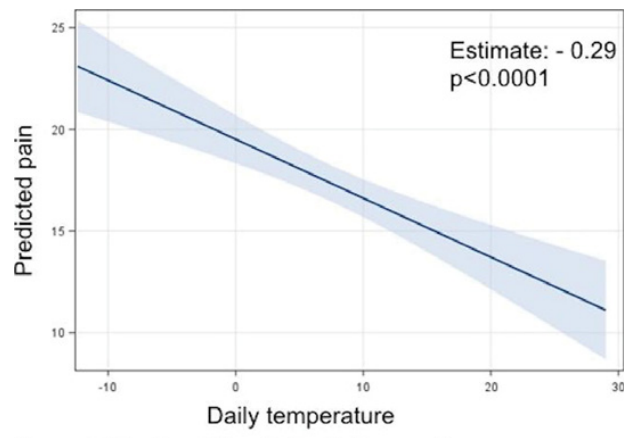

Figure 1. Mixed model analysis of influence of temperature on pain of patients demonstrating sensitivity to cold temperature

Conclusions: Our results indicate that a subgroup of patients with RA show significant sensitivity to cold temperature, and that these patients are characterized by higher pain and PGA levels at lower daily temperatures. These aspects may have to be taken into account in longitudinal analyses of disease activity of RA.

Disclosure of Interest: None declared

DOI: 10.1136/annrheumdis-2017-eular.5134

\section{THU0634 COMPARING PREFERENCES OF PATIENTS WITH RHEUMATIC DISEASES, OF RHEUMATOLOGISTS, NURSES AND PHARMACISTS TOWARD THE TREATMENT OF RHEUMATIC DISEASES WITH BIOLOGICAL AGENTS: RESULTS FROM THE CARA STUDY}

P. Sarzi-Puttini ${ }^{1}$, P.A. Cortesi ${ }^{2,3}$, L. Sinigaglia ${ }^{4}$, C.M. Montecucco ${ }^{5}$, R. Giacomelli ${ }^{6}$, G. Lapadula ${ }^{7}$, I. Olivieri ${ }^{8}$, A. Giardino ${ }^{9}$, G. DIdoni $^{10}$ L. Scalone ${ }^{2,3}$, L.G. Mantovani ${ }^{3,11}$, M. Mecchia ${ }^{10}$ on behalf of CARA Working group. ${ }^{1}$ Rheumatology Unit, L. Sacco University Hospital, Milano; ${ }^{2}$ Univeristy of Milano-Bicocca, Monza; ${ }^{3}$ CHARTA Foundation; ${ }^{4}$ Rheumatology Unit, G. Pini Hospital, Italy, Milano; ${ }^{5}$ University of Pavia School of Medicine, IRCCS Policlinico San Matteo Foundation, Pavia; ${ }^{6}$ Rheumatology Unit School of Medicine, University of L'Aquila, L'Aquila; ${ }^{7}$ Rheumatology Unit, University of Bari, Bari; ${ }^{8}$ San Carlo Hospital of Potenza and Madonna delle Grazie Hospital of Matera, and the Basilicata Ricerca Biomedica (BRB) Foundation, Potenza and Matera ${ }^{9}$ MSD; ${ }^{10}$ MSD Italia, Roma; ${ }^{11}$ University of Milano Bicocca, Monza, Italy

Background: The management of patients with rheumatologic diseases (RDs) involves a complex interaction between different parties such as patients, physicians, pharmacists. The development of biologic therapies has created a more complex decision-making process to select the treatment option for patients. To optimize the appropriateness of the decisions, it is necessary to be informed and aware of the preferences of the interested parties.

Objectives: To estimate preferences of relevant treatment characteristics with biological agents valued by the different subjects involved in the management of patients with rheumatic diseases.

Methods: We involved patients with RDs, rheumatologists, nurses and pharmacists with experience in the treatment with/provision of biological drugs of these patients. Through a Discrete-Choice-Experiment, the participants valued 16 possible scenarios in which pairs of similarly effective treatments were described with 6 characteristics including 2-4 possible levels each: (1) frequency of administration; (2) mode and place of administration; (3) hospitality, service, efficiency and courtesy of health personnel; (4) frequency of reactions at the site of drug administration; (5) generalized undesired/allergic reactions; (6) additional cost: since the Italian NHS pays treatment costs, we included possible additional cost as monthly healthcare taxes for all the citizens, to make available the treatment to all target patients. relative importance of each characteristic was estimated through a random-effects conditional logistic regression model.

Results: 513 patients, 110 rheumatologists, 51 nurses and 46 pharmacists from 30 centres through Italy participated. Characteristics (3), (4) and (6) were the most important for every subgroup, while (1) was the least important for patients and rheumatologists, (2) the least important for the pharmacists, (2) and (5) the least important for the nurses. The four subgroups were consistent in preferences towards the specified levels of characteristics (1) and (3) to (6). However, as for characteristic (2), pharmacists preferred autonomous subcutaneous injection with syringe, nurses preferred assisted infusion at an infusional center close to patient's place, patients and rheumatologists preferred autonomous subcutaneous injection with pen.

Conclusions: Different subjects show different preferences for some treatment characteristics, which together with pure clinical aspects can play an important role in the choice and consequent success of treatments.

Disclosure of Interest: P. Sarzi-Puttini: None declared, P. Cortesi Grant/research support from: Gilead, L. Sinigaglia: None declared, C. Montecucco: None declared, R. Giacomelli Grant/research support from: MSD, G. Lapadula: None declared, I. Olivieri: None declared, A. Giardino Employee of: MSD Italia, G. DIdoni Employee of: MSD Italia, L. Scalone: None declared, L. Mantovani: None declared, M. Mecchia Employee of: MSD Italia

DOI: 10.1136/annrheumdis-2017-eular.4833

\section{THU0635 PRECISE AND SIMPLE ALGORITHM TO IDENTIFY RA CASES} IN ELECTRONIC MEDICAL RECORDS

R. Knevel ${ }^{1,2,3,4}$, S. Raychaudhuri ${ }^{1,2,4}$, K. Liao $^{1,2}$, T.W. Huizinga ${ }^{3}$.

${ }^{1}$ Rheumatology, Brigham and Women's Hospital, Boston: ${ }^{2}$ Harvard Medical School, Cambridge, United States; ${ }^{3}$ Rheumatology, Leids Universitair Medisch Centrum, Leiden, Netherlands; ${ }^{4}$ Broad Institute, Cambridge, United States

Background: Electronic medical records (EMR) have emerged as a large-scale data collection option for observational studies. These huge data registries create new opportunities to study the rheumatologic phenotype and the real-life implications for these diseases. EMR usage poses new questions of quality management, such as how to reliably identify patients with the disease or phenotype of interest, as well as bioinformatic tools to handle the magnitude of data.

Thusfar, the algorithms used to identify cases are often not validated and overly simplify by using one financial code, or they are well-validated but require very specific information which hampers the applicability to other datasets.

Objectives: Aim I: Test the accuracy of the identification of patients with rheumatoid arthritis (RA) using the financial coding system.

Aim II: Develop a simple and precise algorithm to select patients with RA that is easy to implement at other centers.

Methods: Aim I: Out of the 16,183 Rheumatology patients in the Leiden out-patient EMR system 400 charts were randomly selected and reviewed for the rheumatologic diagnosis. Next, the charts were reviewed for 200 randomly selected patients that were labeled as RA in the financial system.

Aim II: To enable generalizability, only codified data that was obtained at regular outpatient clinic visits was used. Lasso regression was applied to identify the most discriminative variables.

Results: Aim I: Since 2008, 16,183 patients were enrolled in the EMR system of the Leiden rheumatology outpatient clinic. 2,845 of these patients were classified as having RA in the financial system. $63 / 400(16.3 \%)$ of the reviewed charts concerned patients with RA. The majority $(n=57)$ were registered as having RA in the financial system. Still, $33 \%$ of the patients with the financial code RA did not have RA.

Aim II: Using Least Absolute Shrinkage and Selection Operator (LASSO) regression anti CCP, MTX prescription and number of visits were identified as the most discriminative variables. Combining these with the presence of the financial code for RA improved the algorithm from an accuracy of $67 \%$ to $90 \%$.

Conclusions: The vast majority of patients that are classified as having RA are registered as such in the financial system. However, a substantial number of patients are registered as RA in the financial system are not classified as RA in clinical charts. Using widely available data on anti-CCP status, MTX prescription 